\title{
Is Federer Stronger in a Tournament Without Nadal? An Evaluation of Odds and Seedings for Wimbledon 2009
}

\author{
Christoph Leitner, Achim Zeileis, Kurt Hornik \\ Vienna University of Economics and Business
}

\begin{abstract}
Wimbledon is one of the most popular annual sports tournament. In the Men's Single 2009 the top seeded and defending champion Rafael Nadal withdrew from the tournament due to injury days prior to the tournament. Here, we try to analyze the effects of Nadal's withdrawal especially on the ability/strength of the main competitor Roger Federer by using bookmakers expectancies to estimate the unknown abilities of the players and compare them for two different odds sets. The comparison shows that the bookmakers did not incorporate Nadal's withdrawal adequately, assigning too high expected winning probabilities to Federer and Murray.
\end{abstract}

Keywords: Abilities, Consensus, Odds, Sports Tournaments, Wimbledon.

\section{Introduction}

The Championships, more commonly known as Wimbledon, is the oldest tennis tournament, being held at the All England Club in the London suburb of Wimbledon since 1877. It is the most popular tournament played on grass in the world and belongs to the four annual major tennis tournaments, the Grand Slams, along with the Australian Open, the French Open and the US Open (see Wimbledon, 2009).

In the Men's singles of Wimbledon 2009 the top seeded and defending champion Rafael Nadal withdrew from the tournament due to injury days prior to the tournament. Here, we analyze the effects of this withdrawal, especially on the expected ability of the bookmakers' favorite Federer. Therefore, we compare different measures of performance, like the official rankings of the Association of Tennis Professionals (ATP), the seeding, and the bookmakers expectancies measured in odds. After showing that the bookmakers odds which are prospective ratings of the participating players' performance perform better, in terms of forecasting the tournament outcome, than the Wimbledon seeding and the ATP ranking, we estimate the abilities of each participating player using two different odds sets including expectancies of a variety of bookmakers: One including winning expectancies for Nadal, and one obtained after his withdrawal. The comparison of the estimated abilities shows that Federer's and Murray's chance of winning Wimbledon 2009 was overestimated by the bookmakers after Nadal's withdrawal. Furthermore, we use all estimated abilities to simulate the outcome of three different tournament designs, showing that in the long run the seeding has not that much influence and a round-robin tournament would be more favorable to top players than the origin single elimination tournament.

In recent literature, ATP rankings as well as seedings, which are based on ATP rankings are used to predict the winner of a tennis match (e.g., Barnett and Clarke, 2005; Klaassen and Magnus, 2003) or a major tennis tournament (e.g., Clarke and Dyte, 2000; Boulier and Stekler, 1999). Bookmakers can be seen as experts in the matter of sports 
(e.g., Forrest and Simmons, 2000) and their odds were successfully used to predict the outcome of single games (e.g., Spann and Skiera, 2009) or European football tournaments (see Leitner, Zeileis, and Hornik, 2009a and Leitner, Zeileis, and Hornik, 2009b).

The remainder of this paper is organized as follows: Section 2 provides a tournament and data description of Wimbledon 2009 for which the players' abilities are modeled and analyzed in Section 3. Section 4 concludes the paper.

\section{Wimbledon 2009: Tournament and Data Description}

\subsection{Tournament}

In the Men's Singles of Wimbledon 2009, a total of 128 international tennis players compete in a single elimination tournament modus (knockout system) to determine the "best" tennis player on grass. Players wishing to enter Wimbledon are required to submit their entry on a special form. The organizing committee evaluates all applications for entry, and use ATP rankings to determine which players will be admitted directly into the tournament, those who have to qualify and those who are rejected. A player without a high enough ATP ranking can be admitted as a "wild card" by the committee. Wild cards are usually offered on the basis of past performance at Wimbledon or to increase British interest. A player who neither has a high enough ranking nor receives a wild card can participate in a qualifying tournament (a three-round event) held one week before Wimbledon. The players who win all three rounds will progress. "Lucky losers" are losers from the final round of qualifying competitions - chosen in order of ATP rankings - to fill any vacancy which occurs in the draw before the first round has been completed. The committee seeds the top 32 players based on their ATP rankings in order to make sure that the top 32 players do not meet each other in the tournament before the third round. The seedings can also be changed due to players' previous grass court performance by the committee (see Wimbledon, 2009).

\subsection{Data}

Bookmakers Odds. Long-term odds of winning Wimbledon 2009 (Men's Singles) were obtained from the website http://odds. bestbetting. com which compares odds of a variety of international bookmakers. We obtained all available odds on two different dates, 2009-06-16 (before the tournament draw and before Nadal's withdrawal; henceforth called W1) and on 2009-06-22 (before the tournament started, but after the draw; henceforth called W2). The first dataset contains odds of 17 international bookmakers for 96 players who are expected to participate in Wimbledon 2009. The latter dataset contains odds of 15 international bookmakers for 105 participating players.

The quoted odds of the bookmakers can be easily transformed into winning probabilities, but they do not represent the true chances that a player will win the tournament, because they include the stake and a profit margin, better known as the "overround" on the "book" (for further details see e.g., Henery, 1999). To recover the underlying beliefs of the bookmakers, we have to adjust the quoted odds by reducing one, the stake, i.e., the 
payment for placing the bet and adjust it by the profit of the bookmaker, the overround (for more details see Leitner et al., 2009a).

This adjustment is done separately for all bookmakers yielding bookmaker-specific overrounds and expected winning probabilities $p_{i, b}$ for each player $i$ and bookmaker $b$ derived from the adjusted odds.

ATP Rankings (Singles). The South African Airways ATP rankings (singles) is based on the players' results (measured in points) at the four Grand Slams, the eight mandatory ATP World Tour Masters 1000 tournaments and the Barclays ATP World Tour Finals of the ranking period, and the best four results from all ATP World Tour 500 tournaments played in the calendar year. We obtained the points assigned to the rankings (henceforth called ATP ratings) from 2009-06-22 from ATP's website for all 128 participating players and for the injured player Rafael Nadal (Association of Tennis Professionals, 2009).

Seeding and Draw for Wimbledon 2009. As described above, the Wimbledon organizing committee seeds the top 32 players of the tournament based on their ATP rankings and their previous grass court performance. We obtained the seeding for Wimbledon 2009 from 2009-06-17 and from 2009-06-19 (after Nadal's withdrawal) from the Wimbledon webpage (Wimbledon, 2009). Additionally, we obtained the draw from 2009-06-19. According to the Wimbledon seeding from 2009-06-17 Nadal was the top seeded player, followed by Federer, Murray, Djokovic, and Del Potro. Due to Nadal's withdrawal after the draw, the committee left the top position blank, and seeded the previously unseeded player Kiefer as 33 and included Thiago Alves as a lucky loser to the draw. The draw changed in that way, that Del Potro (seeded on 5) took the place from Nadal, Blake seeded as 17 took Del Potro's place, and Kiefer took Blake's place.

\section{Modeling Players' Abilities}

The focus of our paper is to analyze the effect of Nadal's withdrawal from Wimbledon 2009, especially on the expected abilities of the main competitor Federer. It is obvious that Nadal's withdrawal increases, on average, the chance of winning the tournament of all other players. However, the ability/strength of each player should not change. Thus, the winning probability for a specific match, e.g., Federer beating Murray in a potential Wimbledon 2009 final, should not be affected by Nadal's withdrawal. The "true" abilities of the players are unknown, but an approximation can be derived from performance measures or winning expectancies, like the ATP rating, the seedings, or the bookmakers odds. Here, we compare all three rating strategies in a forecasting study for Wimbledon 2009. As in previous studies (e.g., Leitner et al., 2009a, 2009b), we find that a consensus derived from the (prospective) bookmakers odds has higher predictive power than retrospective ratings based on historical results (in this study, the Wimbledon seeding and the ATP rankings, see Table 2). Subsequently, we estimate players' abilities based on bookmakers odds using two different odds sets: one including winning expectancies for Nadal and one obtained after his withdrawal. The resulting expected abilities are compared to assess 
Table 1: Estimated winning probabilities $\widehat{p}_{i}$, their associated winning $\left.\operatorname{logits} \widehat{\operatorname{logit}\left(p_{i}\right.}\right)$, estimated $\log$-abilities $\log \left(\right.$ ability $\left._{i}\right)$ and associated simulated winning probabilities $\widetilde{p}_{i}$ of the top ten participating players of Wimbledon 2009 and Nadal using their winning odds from 2009-06-16 (W1) and from 2009-06-22 (W2).

\begin{tabular}{l|rrrr|rrrr}
\hline & \multicolumn{2}{|c}{$\widehat{p}_{i}(\%)$} & \multicolumn{2}{c|}{$\widehat{\operatorname{logit}\left(p_{i}\right)}$} & \multicolumn{2}{c}{$\log \left(\right.$ ability $\left._{i}\right)$} & \multicolumn{2}{c}{$\widetilde{p}_{i}(\%)$} \\
& $\mathrm{W} 1$ & $\mathrm{~W} 2$ & $\mathrm{~W} 1$ & $\mathrm{~W} 2$ & $\mathrm{~W} 1$ & $\mathrm{~W} 2$ & $\mathrm{~W} 1$ & $\mathrm{~W} 2$ \\
\hline Federer & 38.52 & 45.95 & -0.468 & -0.162 & -3.627 & -3.315 & 38.68 & 46.17 \\
Murray & 18.31 & 23.00 & -1.496 & -1.208 & -4.409 & -4.030 & 18.50 & 23.04 \\
Nadal & 14.19 & & -1.800 & & -4.492 & & 14.40 & \\
Djokovic & 5.94 & 5.68 & -2.762 & -2.810 & -4.672 & -4.754 & 6.09 & 5.84 \\
Roddick & 2.53 & 3.30 & -3.652 & -3.377 & -5.048 & -4.881 & 2.62 & 3.50 \\
Del Potro & 2.74 & 3.03 & -3.568 & -3.467 & -5.072 & -4.909 & 2.96 & 3.29 \\
Tsonga & 3.33 & 3.01 & -3.369 & -3.472 & -4.905 & -4.839 & 3.49 & 3.16 \\
Söderling & 1.84 & 1.29 & -3.976 & -4.341 & -5.066 & -5.069 & 2.04 & 1.42 \\
Verdasco & 1.43 & 1.22 & -4.231 & -4.398 & -5.340 & -5.226 & 1.61 & 1.38 \\
Haas & 0.81 & 1.12 & -4.810 & -4.485 & -5.653 & -5.327 & 1.01 & 1.27 \\
Hewitt & 0.43 & 0.78 & -5.441 & -4.842 & -5.380 & -5.303 & 0.62 & 0.92 \\
\hline
\end{tabular}

the effect on Nadal's withdrawal. Furthermore, we use the players' abilities in order to compare different tournament designs in a simulation study.

\subsection{Consensus Information}

Since the bookmakers' expectations about Wimbledon 2009 are rather homogeneous, we use a very straightforward aggregation strategy computing the means of the winning logits (i.e., winning log-odds) to find appropriate consensus measures of all bookmakers:

$$
\left.\widehat{\operatorname{logit}\left(p_{i}\right.}\right)=\frac{1}{B} \sum_{b=1}^{B} \operatorname{logit}\left(p_{i, b}\right),
$$

where $B$ is the number of bookmakers and call this strategy bookmaker consensus model (BCM). See Leitner et al. (2009b) for an exploration of several other aggregation strategies including different variance specifications. Transforming these consensus winning logits back to the probability scale yields the bookmakers' consensus winning probabilities $\widehat{p}_{i}$ for each player $i$ for whom odds are available.

Table 1 shows the estimated winning probabilities $\widehat{p}_{i}$ and their associated winning logits $\left.\widehat{\operatorname{logit}\left(p_{i}\right.}\right)$ of the top ten participating players of Wimbledon 2009 using the winning odds W1 and W2. According to the BCM for W1 and W2, Federer has the highest chance of winning Wimbledon 2009 (W1: 38.52\%, and W2: 45.95\%), followed by Murray with a clear distance (W1: $18.31 \%$, and W2: $23.00 \%$ ). The top seeded and number one ATP player Nadal has a rather low expected winning probability of $14.19 \%$ (W1), presumably due to his injury-related cancellation of his participation in the Wimbledon warm-up tournament Queens 2009. Nadal's withdrawal increases the winning probabilities of both players strongly, whereas the winning probabilities of all other players do not change as 
Table 2: Spearman's rank correlation between the actual tournament ranking and rankings according to the estimated BCM winning probabilities, the seeding, and the ATP rating of the top ten participating players of Wimbledon 2009.

\begin{tabular}{lrrr}
\hline & BCM & Seeding & ATP \\
\hline Tournament ranking & 0.109 & -0.156 & -0.185 \\
BCM & & 0.688 & 0.792 \\
Seeding & & & 0.956 \\
\hline
\end{tabular}

Table 3: Correctly prediction of the last 16, 8, 4, 2, and the winner using the (log-)abilities, the seeding, and the ATP ranking of the top 128 participating players of Wimbledon 2009.

\begin{tabular}{lrrrrr}
\hline & \multicolumn{6}{c}{ Round of last ... } \\
& 16 & 8 & 4 & 2 & 1 \\
\hline BCM & 9 & 5 & 3 & 1 & 1 \\
Seeding & 7 & 4 & 2 & 1 & 1 \\
ATP & 8 & 4 & 2 & 1 & 1 \\
\hline
\end{tabular}

clearly. Standard errors for the winning logits are rather low because of the high agreement between the bookmakers, especially for top players, mostly taking values between 0.132 and 0.177 (interquartile range).

In order to test the predictive power of the bookmaker consensus we compare the consensus winning logits including the last available information (W2) with the actual tournament outcome, the Wimbledon seeding, and the ATP ranking of the top ten players using Spearman's rank correlation (Table 2). Although the correlation between the bookmaker consensus winning probabilities and the actual tournament outcome is rather low (0.109) the BCM still performs better than the Wimbledon seeding $(-0.156)$ and the ATP ranking $(-0.185)$. Both, the seeding and the ATP ranking have a negative Spearman's rank correlation with the actual tournament outcome, assigning rather high ranks to two players who reach the quarter-finals (Hewitt) or the semi-finals (Haas).

In addition to the correlation, we analyze the correctly predicted participants of each round (third round to winner). Table 3 shows that the BCM correctly predicts nine players of the last 16, whereas the Wimbledon seeding predicts only seven and the ATP ranking only eight players correctly. Furthermore, the BCM correctly predicts five of the last eight and three of the last four, everytime one more than the Wimbledon seeding and the ATP ranking. All three approaches forecast the actual winner Federer correctly, but expected Murray who was beaten by Roddick in the semi-finals, as the runner-up.

Nevertheless, the ex post analysis shows that the correlation between the bookmakers expectancies for Wimbledon 2009 and the actual tournament outcome is not high, but the bookmakers perform better than the Wimbledon seeding and the ATP ranking. The reasons for the difficulties in forecasting tennis are twofold. First, tennis is an individual sport competition and the outcome of a match/tournament depend only on one individual who can easily have a day off or an injury rather than a whole team. Second, in the tennis tournament design (single elimination tournament) every single match is important, if a 
player loses one match he is eliminated from the tournament.

\subsection{Estimation of Abilities}

With the winning logits and associated winning probabilities we have computed measures for the specific tournament, Wimbledon 2009, including information about the tournament design (in W1 and W2) and including the original draw (in W2). In order to obtain measures of the unknown "true" abilities of the players we have to adjust the winning logits by the tournament effects (tournament schedule and draw). I.e., we try to estimate the abilities which correspond to the winning logits. For this we employ the well known Bradley and Terry (1952) model which measures abilities on a ratio scale and for which the probability $\pi_{i, j}$ for competitor $i$ beating competitor $j$ is given by:

$$
\pi_{i, j}=\frac{\text { ability }_{i}}{\text { ability }_{i}+\text { ability }_{j}} \quad(i \neq j),
$$

where ability $_{i}$ is the ability for competitor $i$.

Thus, if the abilities of all players and the tournament schedule were known, we could easily compute the pairwise probabilities from Equation 2, subsequently simulate a large number of tournament runs (100000 say), and then assess the empirical winning proportions $\widetilde{p}$ for each competitor:

\begin{tabular}{|c|c|c|c|c|c|c|}
\hline $\begin{array}{l}\text { ability } \\
\text { abilities of all } \\
\text { competitors }\end{array}$ & $\rightarrow$ & $\begin{array}{l}\pi_{i, j} \\
\text { pairwise } \\
\text { winning prob- } \\
\text { abilities for all } \\
\text { matches }\end{array}$ & $\rightarrow$ & $\begin{array}{l}100000 \text { runs } \\
\text { tournament } \\
\text { simulations }\end{array}$ & $\rightarrow$ & $\begin{array}{l}\widetilde{p} \\
\text { simulated win- } \\
\text { ning probabili- } \\
\text { ties for tourna- } \\
\text { ment }\end{array}$ \\
\hline
\end{tabular}

However, the vector ability $_{i}(i=1, \ldots, 128)$ is unknown but can be chosen so that the corresponding $\widetilde{p}(\text { ability })_{i}$ matches the BCM winning probabilities $p_{i}$ as closely as possible. In our case, we minimize the total absolute deviation between $\hat{p}$ and $\widetilde{p}$, i.e., using a local search strategy we solve the optimization problem:

$$
\text { ability }=\underset{\text { ability }}{\operatorname{argmin}} \sum_{i=1}^{n} \mid \hat{p}_{i}-\widetilde{p}(\text { ability })_{i} \mid .
$$

In order to simulate the tournament and estimate the ability for each player, winning logits for all players are required. However, these are only available for those players for which odds are provided by at least one bookmaker. Therefore, we impute the missing values using a simple linear regression model relating the ATP ratings (available for all players) on the log-scale to the consensus winning logits by least squares

$$
\operatorname{logit}\left(p_{i}\right)=\beta_{0}+\beta_{1} \cdot \log (A T P) .
$$

In both relationships (W1 and W2) the correlation is rather high (W1: 0.828, W2: 0.836) yielding the slope $\beta_{1}$ (W1: 1.71, W2: 1.73) and the intercept $\beta_{0}$ (W1: $-18.66, \mathrm{~W} 2$ : -18.79. Using these estimated model parameters the consensus winning logits of the 


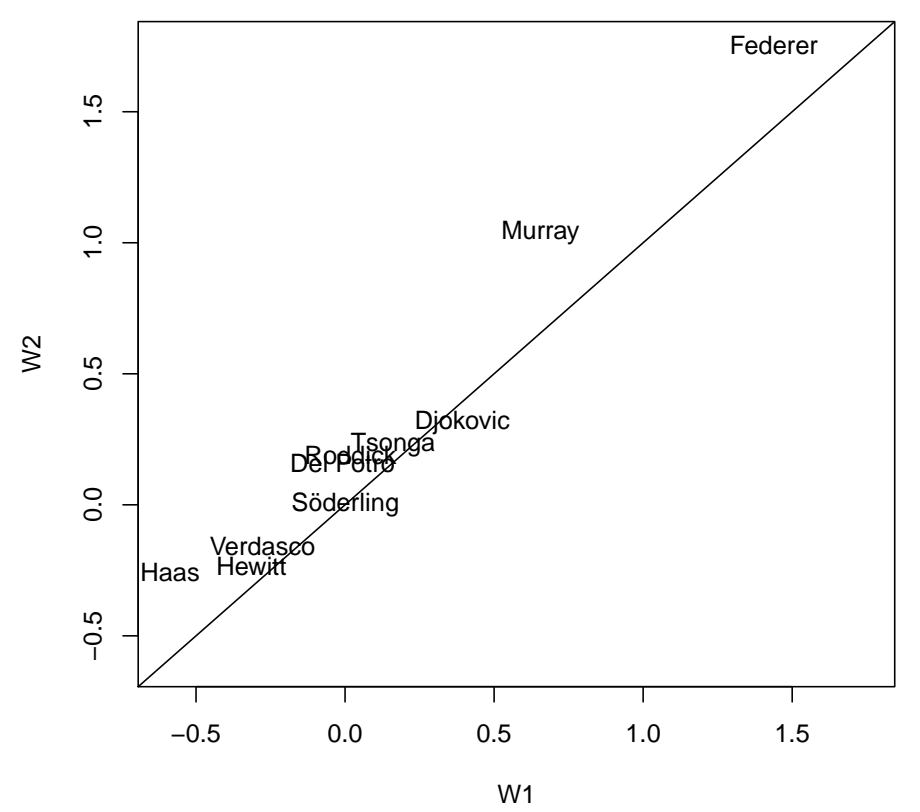

Figure 1: Comparison of the estimated $\log$-abilities $\log \left(\right.$ ability $\left._{i}\right)$ of the top ten participating players of Wimbledon 2009 relative to the log-ability of Söderling using the winning odds from 2009-06-16 (W1) and from 2009-06-22 (W2).

"unrated" players can be predicted. Other imputation strategies would be conceivable, however, the effect on the simulation outcome is rather low because all "unrated" players have rather low ATP ratings and hence low expected winning probabilities: The best "unrated" player is Petzschner at ATP rank 55. For ease of comparison, we show the estimated abilities on the log-scale and their associated simulated winning probabilities $\widetilde{p}_{i}$ (which match the winning probabilities $\widehat{p}_{i}$ derived from the BCM) of the top players of Wimbledon 2009 for $\mathrm{W} 1$ and W2 in Table 1. According to the estimated log-abilities Federer is still the best player of Wimbledon 2009 (W1: -3.627, W2: -3.315), followed again by Murray (W1:-4.409, W2: - 4.030). If Nadal had played Wimbledon 2009, he was expected to be the third strongest player of the tournament (W1: -4.492 , with an associated simulated winning probability of $14.40 \%$ ).

In order to assess whether the ability of a player was altered due to Nadal's withdrawal, we compare the players estimated relative log-abilities, i.e., the log-ability centered by the log-ability of a reference player. Figure 1 depicts these relative log-abilities for the top ten players relative to Söderling who was employed as the reference because his log-abilities for $\mathrm{W} 1$ and $\mathrm{W} 2$ are rather similar. The comparison of the W1 and W2 log-abilities shows that the relative abilities of all top ten players except Djokovic increase. However, there is a marked increase only for Federer, Murray, and Haas, e.g., the probability that Federer beats Söderling increases from $80.84 \%$ to $85.25 \%$.

The changes in the (log-)abilities of Federer, Murray, and Haas show that the book- 
makers do not react to Nadal's withdrawal and its consequential changes of the draw as expected because the winning probabilities before and after the withdrawal are inconsistent. Apparently, they have not considered the whole tournament again and instead just increased the winning probabilities, especially of the top two players. However, this may also be a reaction to a change in the punters' betting behavior, who might bet much more on a tournament victory of these three players. In the case of Murray this could be emphasized by national sentiment of British punters, and in case of Haas by his clear win over Djokovic (6-3 6-7(4) 6-1) in the final of the Wimbledon warm-up tournament in Halle. In any case, both explanations for the changes seem to be far more plausible than interpreting the results literally as an increase in their abilities. In the latter case, one would have to argue that Federer, Murray, and Haas are so relieved by the drop-out of Nadal that they even play stronger in matches against other players (such as Söderling).

\subsection{Effects of the Tournament Design}

With the estimated abilities of the players a measure adjusted for the tournament effects is now available and we are able to determine the effects of different tournament designs by simulating winning probabilities of all participants. A tennis tournament is typically a single elimination tournament and so each match plays an important role. A player with the ambition of winning the tournament is not able to have a day off. Furthermore, in a tennis tournament like Wimbledon a specific number of players is seeded.

In order to determine the effects of the tennis tournament with its seeding, we compare three different designs: (1) a single elimination tournament with the original seeding and draw of Wimbledon 2009, (2) a single elimination tournament without seeding and random draw, and (3) a round-robin tournament, where each player plays each other once. We use the estimated abilities from all 128 players of Wimbledon 2009 derived from the BCM (W2) and simulate their chances of winning the tournament (again using 100000 runs). The resulting winning logits for the top ten players are compared in Figure 2. The winning logits of the single elimination tournament with and without seeding differ not really much. Only some winning logits change slightly (e.g., the logits of Murray and Djokovic slightly decrease) if the single elimination tournament is played without seeding. However, overall these differences are minor signalling that in the long run, the seeding does not have a large effect on the tournament outcome. In contrast, if we consider a round-robin where instead of 127 matches 8128 matches have to be played, the winning probability of the player with the highest ability (here: Federer) increases strongly compared to the single elimination tournaments. The winning logits of all other players (except the second strongest player Murray) decrease sharply. Thus, although being more favorable for the top players, we can conclude that a round-robin tournament would be less exciting than single eliminations. Also, players would have to play 127 matches instead of at most seven matches in single elimination tournaments. 


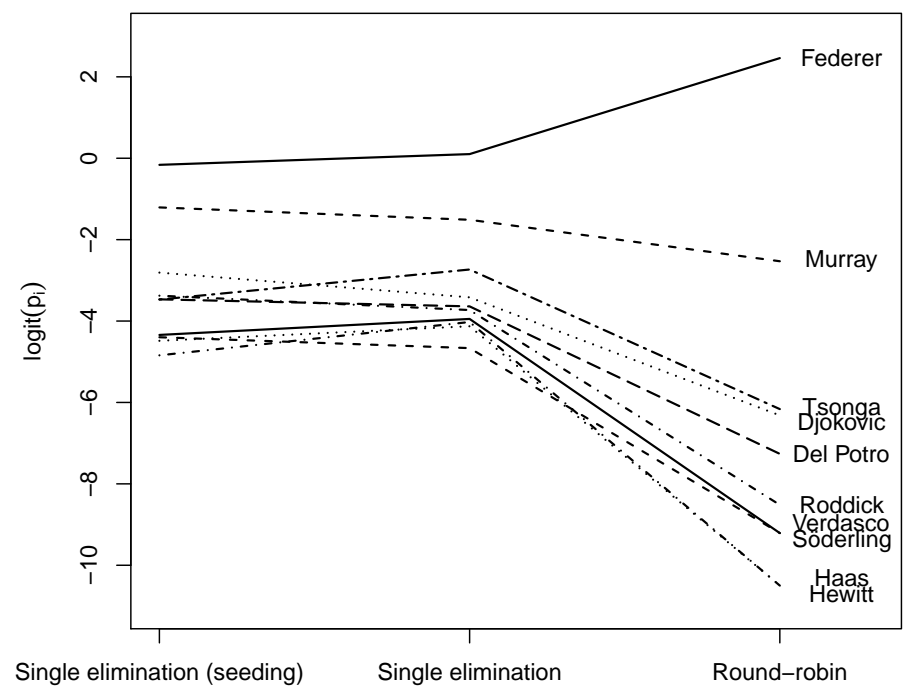

Figure 2: Winning logits of the top ten players simulated by three different tournament designs (single elimination with seeding, single elimination without seeding, and a roundrobin tournament) based on the estimated abilities for all 128 players (W2).

\section{Conclusion}

In this paper we investigate strategies for estimating the expected players' abilities of a tennis tournament (Wimbledon 2009) using bookmakers expectancies for winning the tournament. A comparison of two datasets incorporating different information about the (expected) participants of the tournament shows that the associated estimated abilities before and after a rapid change of the tournament (here: Nadal's withdrawal) are not consistent. More specifically, the abilities of the main competitors (Federer and Murray) as well as the winner of a warm-up tournament (Haas who won the tournament in Halle) increase relative to other top players. An explanation for this could be that the bookmakers did not consider the whole tournament again after the rapid change and simply adjusted some of their odds. This in turn could be a reaction to a change of punters' behavior who bet much more on a few exposed players (possibly emphasized by national sentiment for Murray and the recent tournament victory of Haas). Finally, we also investigate the effect of the tournament schedule on top players' chances of winning the tournament by a simulation study. Three different tournament designs are compared showing that in the long run the seeding of the tournament has only little effect on its outcome.

\section{Computational Details}

All computations were carried out in the R system (version 2.9.2) for statistical computing (R Development Core Team, 2009). 


\section{References}

Association of Tennis Professionals. (2009). Tennis ATP World Tour. (Online: http:// www . atpworldtour . com/; accessed 2009-06-22)

Barnett, T., and Clarke, S. R. (2005). Combining player statistics to predict outcomes of tennis mathces. IMA Journal of Management Mathematics, 16, 113-120.

Boulier, B. L., and Stekler, H. O. (1999). Are sports seedings good predictors?: An evaluation. International Journal of Forecasting, 15, 83-91.

Bradley, R. A., and Terry, M. E. (1952). Rank analysis of incomplete block designs: I. The method of paired comparisons. Biometrika, 39, 324-345.

Clarke, S. R., and Dyte, D. (2000). Using official ratings to simulate major tennis tournaments. International Transactions in Operational Research, 7, 585-594.

Forrest, D., and Simmons, R. (2000). Forecasting sport: The behaviour and performance of football tipsters. International Journal of Forecasting, 16, 317-331.

Henery, R. J. (1999). Measures of over-round in performance index betting. Journal of the Royal Statistical Society D, 48(3), 435-439.

Klaassen, F., and Magnus, J. R. (2003). Forecasting the winner of a tennis match. European Journal of Operational Research, 148(2), 257-267. Available from http://econpapers.repec.org/RePEc: eee:ejores:v:148:y:2003:i: $2: p: 257-267$

Leitner, C., Zeileis, A., and Hornik, K. (2009a). Forecasting sports tournaments by ratings of (prob)abilities: A comparison for the EURO 2008. International Journal of Forecasting. (Forthcoming)

Leitner, C., Zeileis, A., and Hornik, K. (2009b). Forecasting the winner of the UEFA Champions League 2008/09. In R. Koning and P. Scarf (Eds.), Proceedings of the 2nd international conference on mathematics in sport - IMA sport 2009 (pp. 9499). (ISBN 979-0-905091-21-1)

R Development Core Team. (2009). R: A language and environment for statistical computing [Computer software manual]. Vienna, Austria. Available from http: //www.R-project.org/ (ISBN 3-900051-07-0)

Spann, M., and Skiera, B. (2009). Sports forecasting: A comparison of the forecast accuracy of prediciton markets, betting odds and tipsters. Journal of Forecasting, $28,55-72$.

Wimbledon. (2009). The Championships, Wimbledon 2009 - Official site. (Online: http: //www . wimbledon. org/; accessed 2009-09-25)

Authors' address:

Christoph Leitner

Department of Finance, Accounting and Statistics

Institute for Statistics and Mathematics

Augasse 2-6

1090 Vienna

E-mail: christoph.leitner@wu.ac.at 